DOI $10.30898 / 1684-1719.2020 .4 .3$

UDC 535.13: 535.326: 535.36: 621.37

\title{
IRREGULAR LIQUID CRYSTAL WAVEGUIDE STRUCTURES: ANALYSIS OF QUASI-STATIONARY FLUCTUATIONS, POWER LOSS AND STATISTICAL PROPERTIES OF IRREGULARITIES
}

\author{
A. A. Egorov ${ }^{1,2}$, A. S. Ayriyan ${ }^{3,4}$, E. A. Ayrjan ${ }^{3}$ \\ ${ }^{1}$ Moscow A.S. Popov Scientific-Technical Society of Radio Engineering, Electronics and \\ Communications, Sretenskii blv., 2, Moscow 101000, Russia \\ ${ }^{2}$ A.M. Prokhorov General Physics Institute, Russian Academy of Sciences, \\ Vavilov str., 38, Moscow 119991, Russia \\ ${ }^{3}$ Joint Institute for Nuclear Research, Joliot-Curie str. 6, Dubna 141980, Russia \\ ${ }^{4}$ A.I. Alikhanyan National Science Laboratory, \\ Alikhanian Brothers St., 2, Yerevan, 0036, Republic of Armenia
}

The paper is received on March 24, 2020

Abstract. Irregular nematic liquid crystal waveguide structures were investigated by the numeric simulation and experimentally. The dependence of attenuation coefficient (optical losses) of the waveguide modes and the effective sizes (correlation radii) of quasi-stationary irregularities of the liquid-crystal layer on the linear polarization of the incident laser radiation and the presence of pulse-periodic electric field were experimentally observed and investigated. An estimate is made of the correlation radii of liquid-crystal waveguide quasi-stationary irregularities. The observed decrease in the attenuation coefficient of the waveguide modes and the effective sizes of irregularities in the liquid-crystal layer, when the external electric field is switched on, explained by the effect of the decrease in the fluctuations correlation radii of the local orientation of the molecules of the liquid crystal. Experimental spectral density function of the statistical quasi-stationary nematic liquid crystal irregularities was restored and relevant statistical parameters are given. The obtained results are undoubtedly important for further research of dynamic processes inside non stationary waveguide liquid crystal layers, both from the theoretical point of view for understanding kinetic processes in the liquid crystals, and with practical, -- in the organization and carrying out suitable experimental researches.

Key words: waveguide, planar lens, laser radiation, liquid crystal, director, fluctuations, irregularities, optofluidics, numerical simulation. 


\section{Introduction}

In the present work, integrated-optical waveguides and thin-layer waveguide lenses formed with the help of the nematic liquid crystal (NLC) [1, 2], including the imposition of an electric field [3-7], are investigated.

Liquid crystals (LC) are a phase state in which some substances under certain conditions (temperature, pressure, concentration in a solution). Liquid crystals simultaneously possess the properties of both liquids (fluidity) and crystals (anisotropy). By structure, liquids are viscous liquids consisting of elongated or diskshaped molecules, in a certain way ordered in the entire volume of this liquid (see fig. 1). Liquid crystals structure is a viscous fluid consisting of molecules of elongated or disk-like shape, definitely arranged in the entire volume of this liquid.

The NLC does not have a layered structure in the initial state; their molecules glide continuously in the direction of their long axes, rotating around them, but retain a long-range orientation order: the long axes are directed along one preferred direction i.e. director.

The basis of most of the electro- and magneto-optical effects specific for LC is the director's (the axis of the predominant direction of molecules of a macroscopic volume of matter) reorientation under the action of a field or a fluid flow [1-7]. A change in the direction of the preferred orientation of the molecules (director) of the liquid crystal under the influence of an external electric (or magnetic) field leads to a significant change in the properties of the LC. This effect, known as the Fredericks effect, underlies numerous applications of liquid crystals and is the subject of ongoing research. The result of reorientation is the change in the optical properties of the medium. This process of reorienting the director (local or taking place throughout the sample as a whole) can be traced in all electro- and magneto-optical effects.

In this paper a well known nematic liquid crystal 4-Cyano-4'-pentylbiphenyl (5CB) was used to create different studied waveguiding structures. Two dimensional structure of the molecule of NLC 4-Cyano-4'-pentylbiphenyl (5CB) is presented in the fig. 1 . 
The relevance and prospects of these studies is due to the need to develop new methods for studying waveguide structures made of the LC. In addition, there is a need to develop new methods for studying the irregularities of liquid-crystal waveguide layers.

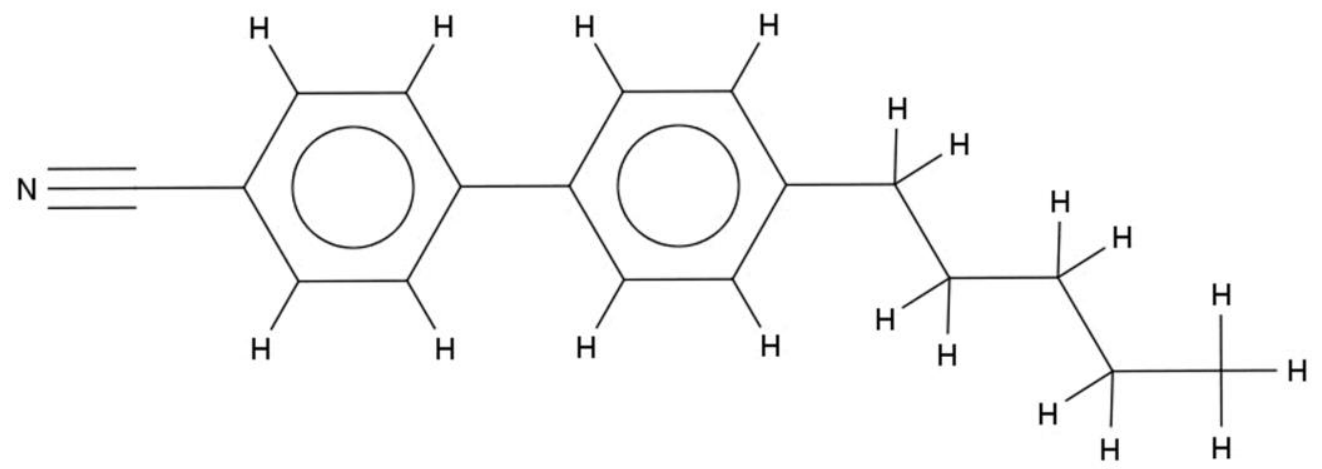

Fig. 1. 2D structure of the molecule of NLC 4-Cyano-4'-pentylbiphenyl (5CB).

An important stimulus to this study is also a need for a waveguide method to investigate and measure physical parameters of NLC layers with a subwave resolution in correlation radii of orientation irregularities [7]. From this point of view the important objective is an improvement and active development of theoretical, experimental, and computer-aided methods for investigation of propagation, transformation, diffraction and scattering of electromagnetic radiation in various irregular waveguiding structures.

\section{Experimental setup. Waveguiding NLC structures}

The objects of the study are the integrated-optical waveguide (fig. 2 and fig. 3) formed from NLC 4-Cyano-4'-pentylbiphenyl (4-Cyano-4'-pentylbiphenyl or 5CB), well known from publications in the scientific and scientific literature (see e.g. [1-5], as well as planar waveguide NLC 3D lenses (fig. 3). In this geometry, the equation of director motion (see [1-4, 6] for details), which expresses the balance between the elastic and viscous torque and the external field torque, has close-to-zero initial and boundary conditions.

In the fig. 2 the following is depicted: 1 is the laser, 2 is the laser beam, 3 is the polarizer, 4 is the lens, 5 is the NLC waveguide, 6 is the waveguide mode 
propagation (in the optical-beam representation), 7 is the vertical profile of the guided TE mode, 8 is schematic representation of LC molecules, 9 is the scattering volume in NLC waveguide, 10 is the laser radiation scattered in NLC waveguide, 11 are glass plates, 12 is the LC layer, 13 is the slit, 14 is the camera, 15 is the computer, 16 is the plotted graph.

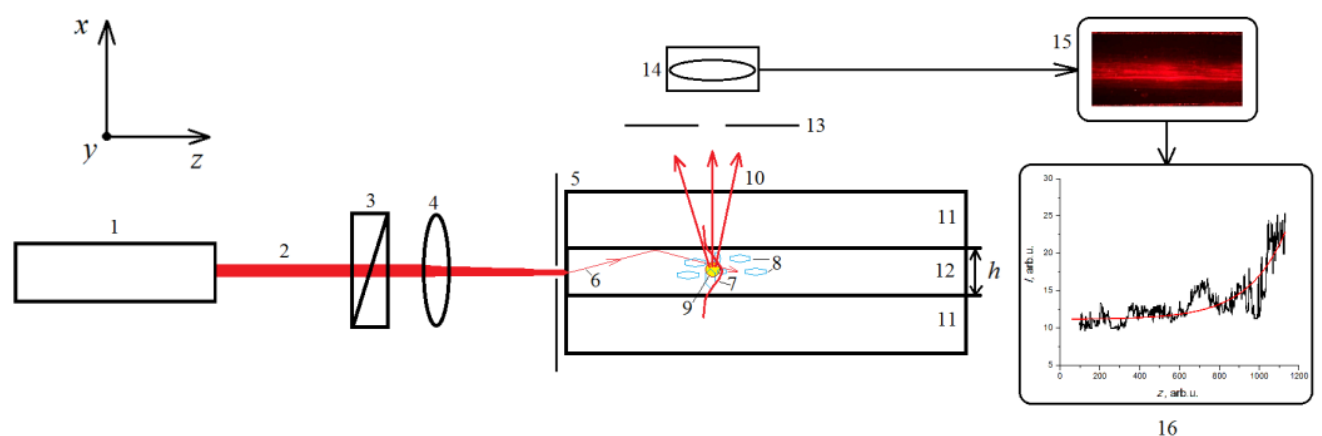

Fig. 2. Schematic representation of the experimental setup.

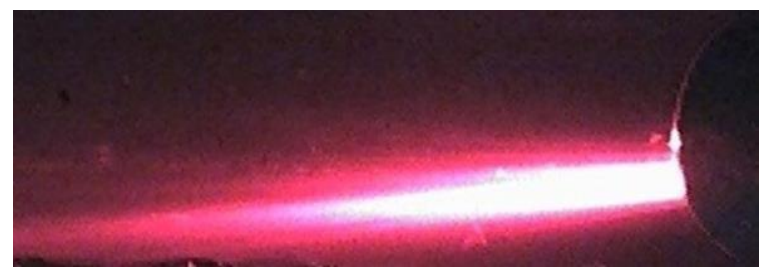

Fig. 3. Photograph of tracks of waveguide modes: left and center of the photo - in the integrated optical NLC waveguide; at the right: the planar 3D NLC lens.

In the fig. 3 the planar NLC lens formed at the end of the waveguide is on the right at the edge of the photograph. Immediately behind the NLC lens, one can clearly see the characteristic beam of radiation focused on it and the tracks of the modes. Dimensions of the area shown in the photo in width are about $3 \mathrm{~mm}$, and in length do not exceed 9-15 mm. Lens dimensions: radius $R$ is $1.5 \mathrm{~mm}$, thickness $h$ in the center was determined by the thickness of NLC waveguides (see below).

\section{Results of the study of NLC structures}

In the experiments multimode NLC waveguides, formed by two glass plates and LC layer between them (fig. 2), were studied. NLC had a homogeneous planar orientation ("poly25" sample) with an optical axis along the direction of the director (coincides with the axis $z$ ). 
Waveguides with different thicknesses $(25,75$, and $125 \mu \mathrm{m})$ were studied in experiments, but the main part of the results is given only for NLC waveguides with $h=25$ and $75 \mu \mathrm{m}$. Distance between copper electrodes was $2 \mathrm{~mm}$. A high-voltage pulse (repetition frequency of about $10 \mathrm{~Hz}$ ) was applied to them. The NLC layer had refractive indices: ordinary $\approx 1.53$ and extraordinary $\approx 1.70$ (for the wavelength of laser radiation $\lambda \approx 0.64 \mu \mathrm{m}$ and temperature $\approx 25^{\circ} \mathrm{C}$ ). The glass plates had refractive indices: $n_{1}=n_{3} \approx 1.52$.

Thus, in experiments and numerical calculations, three-layer symmetric waveguides with the following refractive indices were studied: $n_{1}=n_{3}=1.52, n_{2}=$ 1.53. The input efficiency of radiation into NLC waveguides did not exceed $35 \%$.

In the fig. 4 3D "color" (grey scale) map surface of the dependence of the scattered laser radiation $I(z, y)$ for TM-polarization since the end of the planar lens $\left(I(z, y)=I_{\max }\right)$ and right behind NLC lens is presented. The field $E$ is switched off. For TE-polarization very similar 3D map surface was obtained. In all figures, except fig. 8 and fig. 9, "1 arb.u." is equal approximately to $3.5 \mu \mathrm{m}$.

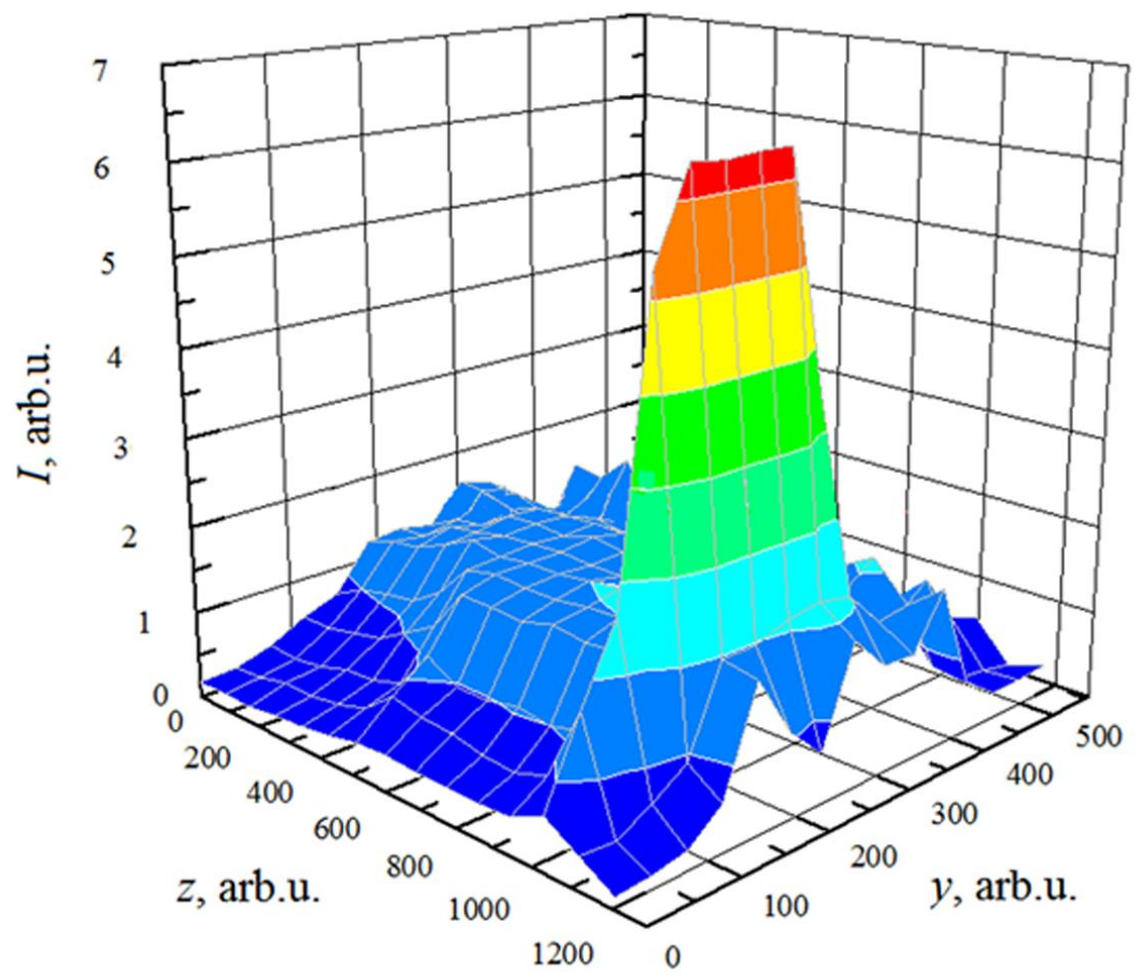

Fig. 4. 3D map surface of the scattered laser radiation on the coordinates $z$ and $y$, since the end of the planar lens and right behind 3D NLC lens: the field $E$ is switched off. TM-polarization of the laser radiation. 
We researched the propagating and scattering of the guided modes on an extended bulk 3D inhomogeneity (insert type) of the nonabsorbing or absorbing waveguide layer. The research three-layer waveguide was formed e.g. by two glass plates and the nematic liquid crystal layer between them (symmetric multimode NLC waveguide), or three-layer waveguide was formed by thin layer of the liquid crystal deposited on the glass substrate (asymmetric multimode NLC waveguide), i.e. the 3D LC waveguide lens was made before the planar waveguide face. Type of the 3D irregularity can be modified by changing the form of the function that sets the inhomogeneity of the waveguide layer, the position of the 3D irregularity in the waveguide, as well as by changing its size.

In order to quantify the optical losses in the waveguide, computer photometry of photographs of the corresponding mode tracks was carried out. To determine the optical losses, we used the well-known expression [6,7]:

$$
I(z)=I_{0} \exp (-\alpha z),
$$

where $I(z)$ is the intensity of radiation (per unit area) at any point along the length of the waveguide, $I_{0}$ is the intensity at $z=0$.

Let's give an example of the results of computer processing of data for some of the researched NLC waveguides both for TE and TM-polarization (see fig. 4, fig. 5, and fig. 6). TE-polarization, without field $E: \bar{\alpha}_{\mathrm{TE}}=4.5 \mathrm{~cm}^{-1}, r_{\mathrm{TE}} \approx 0.15-0.6 \mu \mathrm{m}$; with the field $E: \bar{\alpha}_{\mathrm{TE}}=3 \mathrm{~cm}^{-1}, r_{\mathrm{TE}} \approx 0.1-0.4 \mu \mathrm{m}$. TM-polarization (see fig. 5), with the field $E: \bar{\alpha}_{\mathrm{TM}}=6 \mathrm{~cm}^{-1}, r_{\mathrm{TM}} \approx 1.0-1.8 \mu \mathrm{m}$; with the field $E: \bar{\alpha}_{\mathrm{TM}}=5 \mathrm{~cm}^{-1}, r_{\mathrm{TM}} \approx 0.5-$ $1.5 \mu \mathrm{m}$.

The size of the NLC irregularities was estimated in accordance with the Rayleigh criterion. Having determined the loss $\alpha$ of the optical power in a waveguide in accordance with formula (1), it is possible to estimate the effective dimensions $r_{e f}$ of the irregularities of the LC layer in accordance with the approximate formula $[6,7]$ :

$$
r_{e f} \approx \lambda\left(\alpha h_{e f}\right)^{1 / 2}(4 \pi)^{-1} C(\theta),
$$

where $C(\theta)$ is the dimensionless correction factor. 
An estimate for a number of NLC waveguides, performed in accordance with the Rayleigh criterion, showed that the dimensions of irregularities in the NLC layer vary in the range from about 0.08 to $2.00 \mu \mathrm{m}$, and the error in the determination does not exceed $15-20 \%$.

To explain the established patterns of light scattering in the NLC waveguides, 3D fluctuations in the local orientation of $\operatorname{LC}$ molecules were considered $[1,2,6,7]$. Fluctuations of the director are accompanied by elastic deformation of the medium, which leads to local random changes in the optical properties of the medium, which cause the scattering of light. In this case, the correlation radius for the director fluctuations depends inversely on the magnitude of the applied field.

The known laws governing the scattering of light in a non-waveguide case are explained on the basis of the consideration of fluctuations in the local orientation of the molecules, i.e. director of NLC $[1,2]$. As a result, the radius of correlations $\xi$ of the director 3D fluctuations can be calculated, which shows the distance at which the order given by the wall (the waveguide substrate) is stored, for example, when the field $E$ is applied (the bigger the field, the order is preserved at a smaller distance from the wall). It can be put in correspondence with the found correlation radius of the irregularities of the NLC waveguide.

In the fig. 5 the dependence of the intensity $I$ of the scattered laser radiation on the coordinate $z$ with the field $E$ switched on. Laser irradiation has TM-polarization. The numbers denote: 1 is the experimental curve, 2 is the fitting exponential curve.

Analyses of the laser radiation tracks in fig. 6 showed an inhomogeneous change in the attenuation coefficient $\alpha$ for both (TE and TM) polarizations with transverse displacement (along the $y$ axis). In neighboring mode tracks $\alpha$ can differ by $\approx 10$ $50 \%$. This indicates a fairly strong heterogeneity of the NLC layers. Under the influence of the external field $E$ complex nonlinear processes of transformation of both the domain structures inside the layers and the characteristics of the transmitted, diffracted and scattered electromagnetic radiation were observed. 


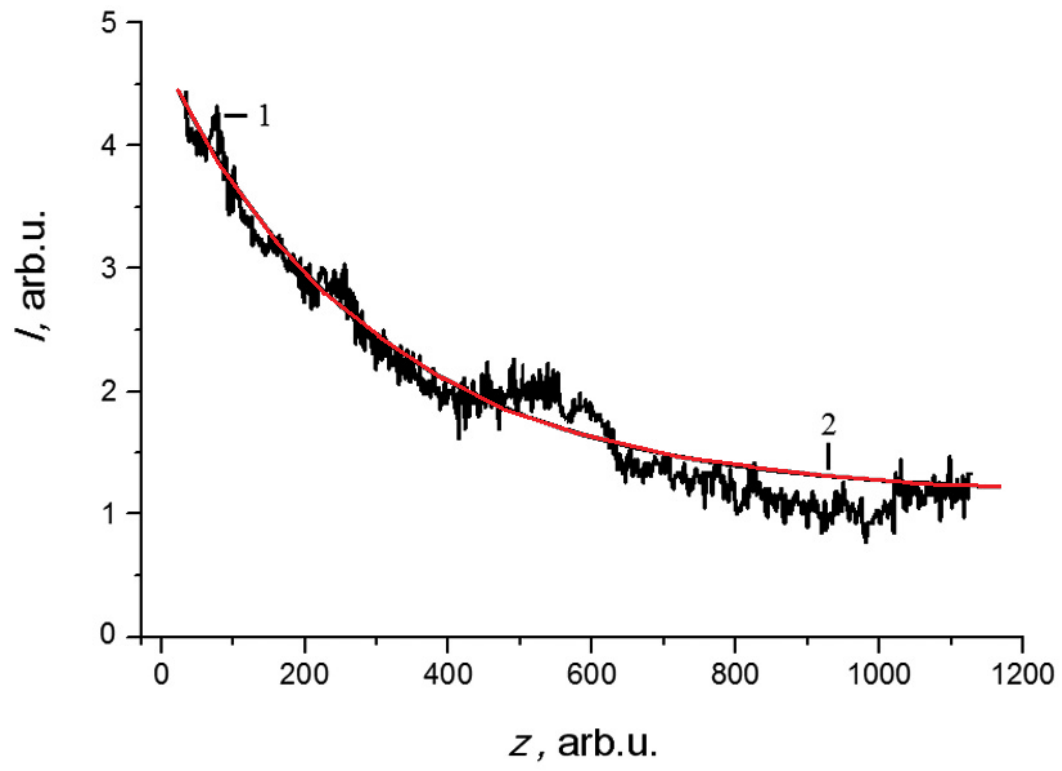

Fig. 5. Dependence of intensity of the scattered laser radiation on the coordinate $z$.

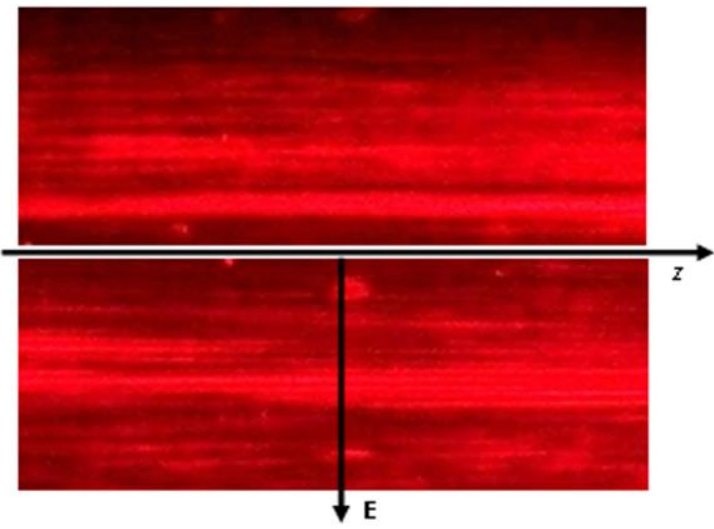

(a)

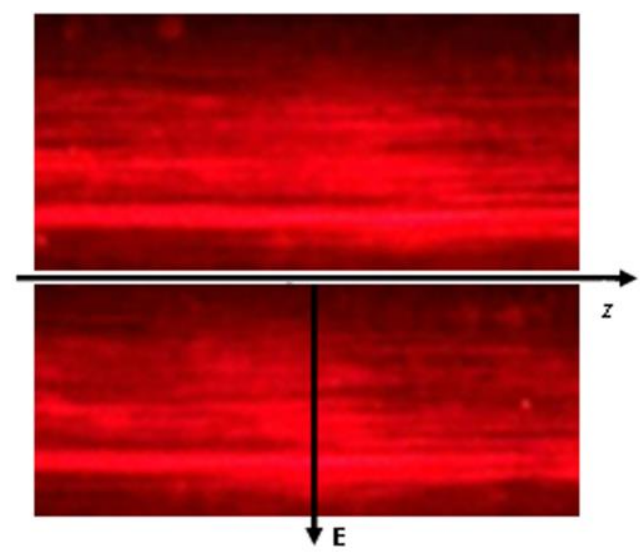

(b)

Fig. 6. Fragments of photo of laser radiation tracks (top view): (a) for TM and (b) for TE-modes in one of the researched integrated optical NLC waveguide. $E$ switched off (on top) and $E$ switched on (below).

Let us compare the regularity of the change in the radius of correlations of the director 3D fluctuations $\xi \propto E^{-1}$ with the results obtained by us for changing the correlation radius of the irregularities of the NLC waveguide without the field and with the field: the correlation radius when the external field is switched on decreases for both types of modes. This indicates a good agreement between our results and the classical theory of director fluctuations in $\operatorname{LC}[1,2,6,7]$. Thus, the obtained estimate confirms the correctness of the experimental dependences found by us. Since 
$I(z) \propto \exp (-\alpha z) \propto \exp \left(-r_{e f} z\right)$, then as the correlation radius $r_{e f}$ decreases, both $I$ and the damping coefficient $\alpha$ decrease, which was discovered in the experiments.

The use of the phenomenon of waveguide $3 \mathrm{D}$ scattering made it possible to substantially increase the resolution by the correlation radii in comparison with the classical methods such as the optical microscopy, interferometry, difractometry and ellipsometry [7-11]. In addition, the waveguide method made it possible to obtain statistical information on waveguide irregularities in one measurement with a sufficiently large volume of the waveguide layer. The advantage of this method is also the possibility of investigating waveguide irregularities in a wide range of changes in their lateral dimensions, including the size of the order of the wavelength of the probing radiation, as in the Mie's scattering theory.

Figure 7 shows one of the radiation intensity profiles measured near the back focal plane of planar NLC lens (curve 1). In the fig. 7: 2 is the curve obtained by smoothing the original distribution 1; 3 is the fitting Gaussian curve; 4 indicates the error (less than 7\%).

The half-width of the distributions of type 1 in the vicinity of the focal plane, measured at the 0.5 level of its maximum (i.e. $\Delta I_{0.5}$ ), reached (10-45) $\lambda$, which is apparently caused by aperture restriction (small numeric aperture $N A$ of the researched LC lenses) and also due to the fact that multimode (14 or more modes) LC waveguides were investigated.

For an example we have compared our measured half-width $\Delta I_{0.5}(y)$ with the half-width intensity profile at the focal distance, including the theoretical diffraction limited spot, given in the paper [5] for tunable liquid crystal lenses.

We can draw a conclusion, that, on the one hand, these sizes $\Delta I_{0.5}$ are close enough, and on the other hand, both of them in several times (>3-6) more than the theoretical diffraction limited spot $\delta[12,13]$ :

$$
\delta=0.61 \frac{\lambda}{N A} \text {. }
$$

In equation (3) numeric aperture $N A$ is usually less than $0.7-1.0$. 


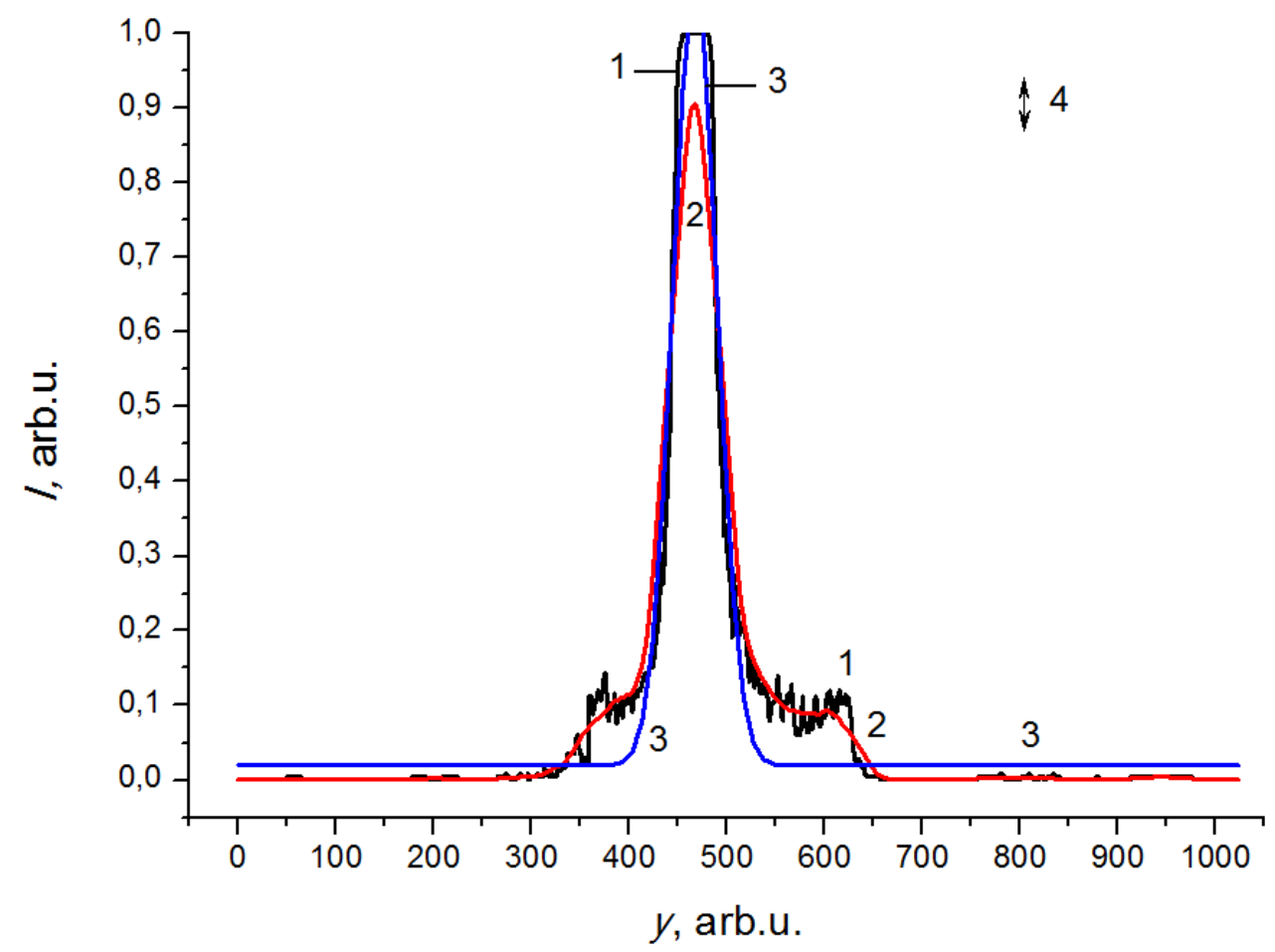

Fig. 7. The intensity profile of laser radiation behind the planar NLC lens.

The estimation of the focal length of the studied liquid-crystal lenses has shown that it is approximately in the range from $2 R$ to $8 R$, where $R$ is the radius of the planar LC lens (see fig. 2).

In the fig. 8 one of the experimental dependences of the scattered laser radiation intensity $I$ on $\gamma$ in the smoothly irregular NLC waveguide at the selected distance $z$ which is measured from the back of the lens is depicted. The range of variation in $y$ from 0 to 1000 approximately corresponds to the range of variation in $\gamma$ from 1.515 to 1.530 , because the data are obtained for the waveguiding nematic liquid crystal lens with variable thickness $h(y, z)$ (see fig. 3 and fig. 4). Fig. 8 shows scattering features of TE-modes in a wide range of slow-down coefficient $\gamma$ variations. Numerical simulation also revealed some complex nonlinear processes of the radiation field transformation $E(x, y, z ; \gamma)$ upon the propagation of the field $E$ from the irregular NLC-glass interface into the environment. This diagram was obtained at a small distance $z$ behind the entrance NLC lens (with a variable thickness $h(y, z)$ close at maximum to the waveguide thickness $h)$ through which 
radiation was input in the liquid-crystal waveguide. Similar results were obtained for the TM polarization. Analogous effects were observed at other NLC waveguide thicknesses.

In the fig. 9 one of the restored Gaussian approximating experimental spectral density function (SDF) $F$ of statistical quasi-stationary irregularities of the NLC waveguide is depicted, where 1 arb.u. along the $y$ axis is about $20 \mu \mathrm{m}^{-1}$.

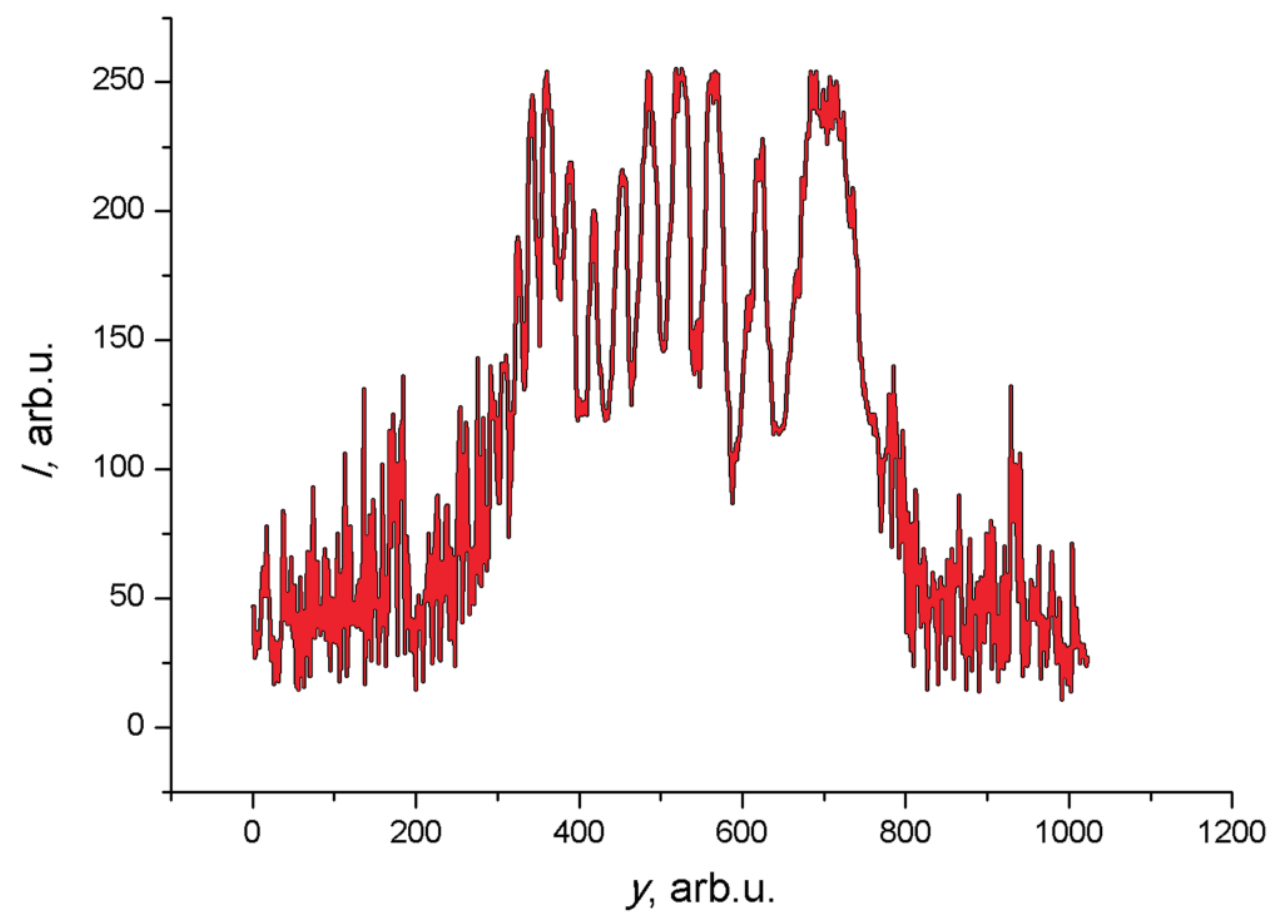

Fig. 8. Experimental dependence of the scattered laser radiation intensity $I$ on $\gamma$ in the smoothly irregular NLC waveguide at $z \approx 3 \mathrm{~mm}$. TE-polarization.

This Gaussian spectral density function $F$ in fig. 8 was obtained by processing the diagram $I$ registered during observation of the mode track from above, i.e. at an angle of 90 degrees to the incident laser radiation. It is obvious that in this case, the main contribution will be made by irregularities from the average size range of $r_{e f}$, which fall into the range of cover modes: $0.5-6 \mu \mathrm{m}$. While considering the possible contribution of leaky modes, this range should be expanded. When the pulse periodic electric field $E$ was switched on one can observed appearance of more large $r_{e f}$. 


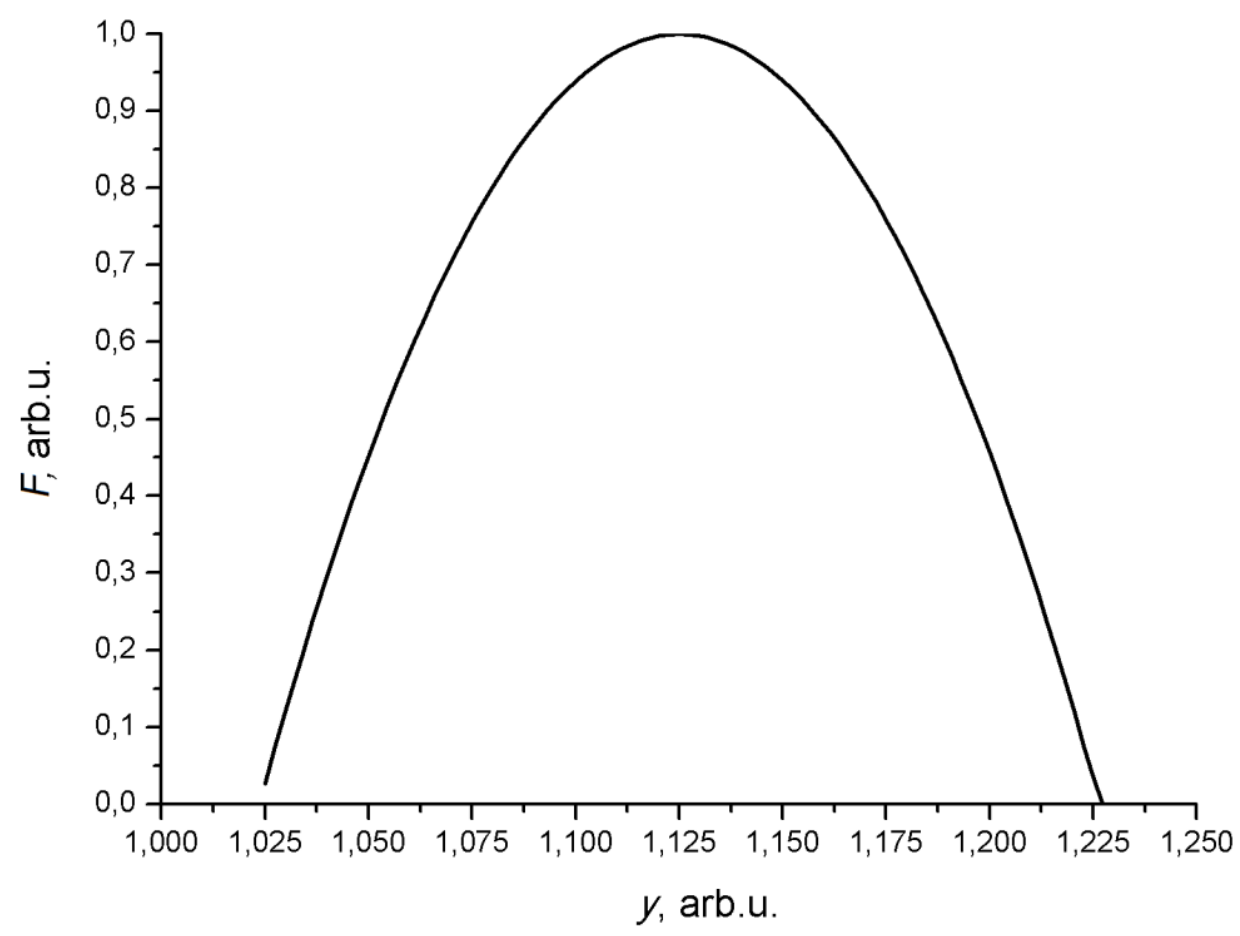

Fig. 9. Experimental Gaussian SDF of the statistical irregularities of the NLC waveguide. TE-polarization. Signal-to-noise ratio $\geq 10$. 1 arb.u. along the $y$ axis is about $20 \mu \mathrm{m}^{-1}$.

Gaussian spectral density function has the form:

$$
F(\beta)=\frac{2 \sigma^{2} r}{L} \exp \left[-\left(\beta_{0}-\beta\right)^{2} r^{2} / 2\right],
$$

where $L$ is the length of the irregular area, $\beta$ is the longitudinal component of the propagation constant of the radiating modes, $\beta_{0}=k \gamma_{0}, k=2 \pi / \lambda, \gamma_{0}$ is the guided mode deceleration coefficient.

The estimation of the possible standard deviation $\sigma$ and $r$ for the experimental Gaussian SDF (see (4)) gives the following result: $\sigma \approx 1-9 \mu \mathrm{m}, r \approx 1-6 \mu \mathrm{m}$ (signalto-noise ratio $\approx 10$ ). When the pulse periodic electric field $E$ was switched on one can observed emergence of more big $\sigma$ and $r$. These outcomes correspond well with the estimates of root-mean-square spatial fluctuations of the director $\sigma_{L p}$ obtained in the paper [7].

We should note that in sufficiently strong external fields, domain structures arise both in the planar and in the homeotropic initial orientation of the NLC. The domain period is always on the order of the layer thickness $h$ (and not the distance between 
the electrodes) [1]. That is observed in our case for the "poly25" sample (planar orientation, $h \approx 25 \mu \mathrm{m}$ ), the size and period of light lines (domains) $\Lambda_{p} \approx 30-80 \mu \mathrm{m}$ for both polarizations. So we can see that in fact: $\Lambda_{p} \propto(1-3) h$. For the homeotropic orientation of the NLC we have: $\Lambda_{h}<\Lambda_{p}$. This indicates a good agreement between our results and classical experiments with nematic liquid crystals.

For TM-polarization similar results were obtained. A more detailed analysis of an inverse waveguide scattering problem and these quasi-stationary effects (due to the director motion) are beyond the scope of this work (see e.g. [6-11]).

To improve the obtained results, especially when irregularities change from small-scale $(\approx 0.1-10 \mu \mathrm{m})$ to large-scale $(\approx 20-100 \mu \mathrm{m})$, one need to use in the numeric analysis the analytic solutions of the problem of waveguide propagation, diffraction and scattering of electromagnetic monochromatic radiation in 3D-irregular and smoothly-irregular waveguides and waveguiding structures [7-11, 14-23].

One and 2D model of Frederiks effect were used for the analysis of the electric field effect on NLC director orientation in the cell that helped us to understand the behavior of the nematic liquid crystal under study (see e.g. [1-3, 4, 6, 7]). Corresponding partial differential equation of the second order can be written in the next form [24]:

$$
4 \mathrm{~K}\left(\theta_{x x}+\theta_{y y}\right)+\frac{1}{2} \varepsilon_{0} \Delta \varepsilon E^{2} \sin 2 \theta=0,
$$

where the constant $K=\left(K_{11}+K_{33}\right) / 2$, and appropriate elastic modules are equal, respectively: $K_{11}=7 \cdot 10^{-12} \mathrm{~N}, K_{33}=10 \cdot 10^{-12} \mathrm{~N} ; \varepsilon_{0}=8,854 \cdot 10^{-12} \mathrm{~F} / \mathrm{m}$ is vacuum electric constant; $\Delta \varepsilon=14$ is dielectric constant anisotropy; $\theta_{\alpha \alpha}=\partial^{2} \theta / \partial \alpha^{2}, \alpha=x, y$.

Frederiks transition threshold for the central part of the cell, as well as dependencies of the distribution of the director orientation on the high electric field have been obtained. The results of the numeric calculations for different cases have been compared to the experiments (see e.g. [3, 4, 6, 24]).

As an example in the fig. $102 \mathrm{D}$ director dynamics in the investigated 5CB NLC cell for the case described by the equation (5) is depicted, where $E \rightarrow 0$, and the 
possible influences of cross members were also taken into account:

$$
\theta_{x} \equiv \frac{\partial \theta}{\partial x}, \theta_{y} \equiv \frac{\partial \theta}{\partial y}, \theta_{x x} \equiv \frac{\partial^{2} \theta}{\partial x^{2}}, \theta_{x y} \equiv \frac{\partial^{2} \theta}{\partial x \partial y}, \theta_{y y} \equiv \frac{\partial^{2} \theta}{\partial y^{2}} .
$$

The boundary conditions were specified as:

$$
\begin{gathered}
\theta(x,-d / 2)=0, \theta(x, d / 2)=0 ; \\
\theta(-L / 2, y)=\pi / 2, \theta(L / 2, y)=\pi / 2,
\end{gathered}
$$

where $d$ is LC layer thickness (i.e. $d=h$ ), $L$ is distance between electrodes, $L=$ $2 \cdot 10^{-3} \mathrm{~m}$.

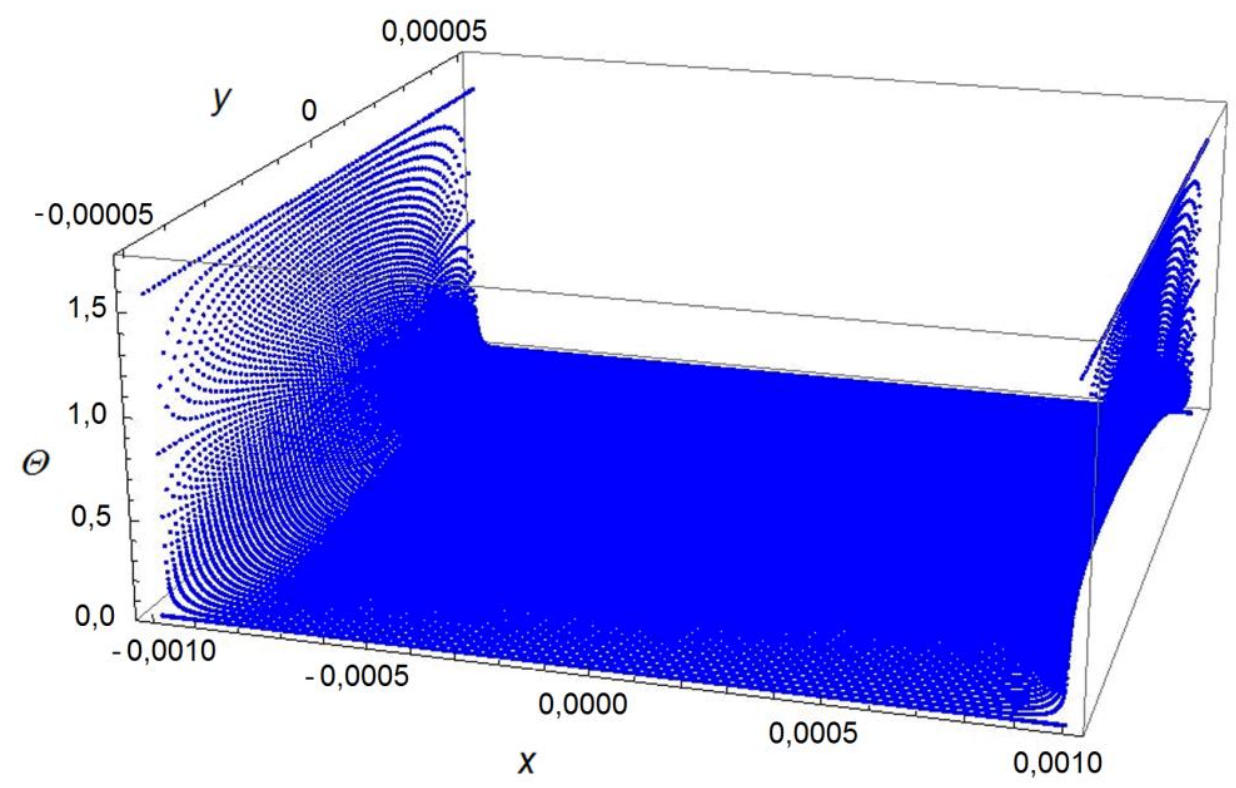

Fig. 10. 2D director dynamics in 5CB NLC cell.

The numeric solutions for equation and its variants (5) were obtained by the standard finite-difference methods. The programmes for numerical solution and computer modeling of two-dimensional parabolic partial differential equation were developed both in FORTRAN, and C/C++ (see e.g. [4-8, 24, 25]).

\section{Conclusion}

Nematic liquid crystal waveguiding structures were researched by the numeric simulation and experimentally. The dependence of the attenuation coefficient of the waveguide modes and the sizes of the quasi-stationary irregularities of the liquidcrystal layer on the linear polarization of the incident laser radiation and the presence 
of a pulse-periodic electric field is experimentally observed. An estimate is made of the correlation radii of liquid-crystal waveguide irregularities. The observed decrease in the damping coefficient of the waveguide modes and the dimensions of irregularities in the liquid-crystal layer, when the external field is switched on, explained by the effect of a decrease in the fluctuations correlation radius of the local orientation of the molecules of the liquid crystal. Restored approximating experimental spectral density function of the statistical irregularities in the irregular NLC waveguide is depicted, relevant statistical parameters are also given, and the problem of non-stationarity of the investigated process is discussed in brief.

The results obtained are important for further investigation of dynamic processes inside non stationary waveguide liquid crystal layers, both from the theoretical point of view for understanding kinetic processes in the liquid crystals, and with practical, - in the organization and carrying out appropriate experimental and theoretical researches in the field of the optofluidics and waveguide optics.

\section{Acknowledgments}

The publication has been funded by RFBR according to the research project: No. 19-01-00645.

The author is grateful to Drs. V.D. Shigorin and I.A. Maslyanitsyn (GPI of RAS) for help in conducting experiments and discussion of a number of aspects at the initial stage of work, and to Drs. I. Marinov and L. Popova for preparation of the NLC cells (Georgi Nadjakov Institute of Solid State Physics, Bulgarian Academy of Sciences, Bulgaria). Our special thanks to Prof. A. Strigazzi (Physics Institute of Condensed Matter and Complex Systems, Politecnico di Torino, Italy) for drawing our attention to the $2 \mathrm{D}$ problem of research in the area of liquid crystals.

\section{References}

1. Blinov L.M. Elktro- $i$ magnetooptika zhidkikh kristallov [Electro- and Magnetooptics of Liquid Crystals]. Moscow: Nauka, 1978. (In Russian)

2. Khoo I.C. Liquid Crystals. 2nd Edition. NY: Wiley, 2007.

3. Ayriyan A.A., Ayrjan E.A., Egorov A.A., Hadjichristov G.B., Marinov Y.G., Maslyanitsyn I.A., Petrov A.G., Pribis J., Popova L., Shigorin V.D., Strigazzi 
A., Torgova S.I. Some Features of Second Harmonic Generation in the Nematic Liquid Crystal 5CB in the Pulsed-Periodic Electric Field. Physics of Wave Phenomena. 2016. Vol. 24. No. 4. P. 259-267.

4. Ayriyan A.A., Ayrjan E.A., Egorov A.A., Dencheva-Zarkova M., Hadjichristov G.B., Marinov Y.G., Maslyanitsyn I.A., Petrov A.G., Popova L., Shigorin V.D., Strigazzi A., Torgova S.I. Modeling of Static Electric Field Effect on Nematic Liquid Crystal Director Orientation in Side-Electrode Cell. EPJ Web of Conferences. 2018, Vol. 173. 03002.

5. Beeckman J., Yang T.-H., Nys I., George J.P., Lin T.-H., Neyts K. Multielectrode tunable liquid crystal lenses with one lithography step. Optics Letters. 2018. Vol. 43. No. 2. P. 271-274.

6. Egorov A.A., Shigorin V.D., Ayriyan A.S., Ayryan E.A. Study of the effect of pulsed-periodic electric field and linearly polarized laser radiation on the properties of liquid-crystal waveguide. Physics of Wave Phenomena. 2018. Vol. 26. No. 2. P. 116-123.

7. Egorov A.A. Study and analysis of light scattering loss in irregular integrated optical waveguides. Physics of Wave Phenomena. 2019. Vol. 27. No. 3. P. 217 228.

8. Egorov A.A. Use of waveguide light scattering for precision measurements of the statistic parameters of irregularities of integrated optical waveguide materials. Opt. Engineering. 2005. Vol. 44. No. 1. P. 014601-1-014601-10.

9. Egorov A.A. Theoretical and numerical analysis of propagation and scattering of eigen- and non-eigen modes of an irregular integrated-optical waveguide. Quantum Electronics. 2012. Vol. 42. No.4. P.337-344.

10. Egorov A.A. Correct investigation of the statistic irregularities of integrated optical waveguides with the use of the waveguide light scattering. Laser Physics Letters. 2004. Vol. 1. No. 8. P. 421-428.

11. Egorov A.A. Numerical investigation of characteristics of laser radiation scattered in an integrated optical waveguide with three-dimensional inhomogeneity. Optics and Spectroscopy. 2012. Vol. 112. No. 2. P.280-290. 
12. Born M., Wolf E. Principles of optics. NY, Pergamon. 1986.

13. Goodman J.W. Introduction to Fourier Optics. NY, McGraw-Hill. 1996.

14. Sodha M.S., Ghatak A.K. Inhomogeneous optical waveguides. London, Plenum. 1977.

15. Hunsperger R.G. Integrated Optics. Theory and Technology. Springer-Verlag, Berlin-Heidelberg. 1984.

16. Marcuse D. Light transmission optics. NY. Van Nostrand Reinhold. 1972.

17. Unger H.-G. Planar optical waveguides and fibres. Oxford, Clarendon. 1977.

18. Snyder A.V., Love J.D. Optical waveguide theory. NY, Chapman and Hall. 1983.

19. Ayryan E.A., Egorov A.A., Sevastianov L.A., Lovetskiy K.P., Sevastyanov A.L. Mathematical modeling of irregular integrated optical waveguides. Lecture Notes in Computer Science. 2012. Vol. 7125. P. 136-147.

20. Falco A.Di., Kehr S.C., Leonhardt U. Luneburg lens in silicon photonics. Optics express. 2011. Vol. 19. No. 6. P. 5156-5162.

21. Yong M. Optics and Lasers. Including Fibers and Optical Waveguides. Springer. 2001.

22. Liu J.-M. Photonic Devices. Cambridge University Press. 2005.

23. Tsang L., Kong J.A., Ding K.-H., Ao C.O. Scattering of Electromagnetic Waves: Numerical Simulations. NY, Wiley. 2001.

24. Ayriyan A.S., Ayrjan E.A., Egorov A.A., Maslyanitsyn I.A., Shigorin V.D. Numerical modeling of the static electric field effect on the director of the nematic liquid crystal director. Mathematical Models and Computer Simulations. 2018. Vol. 10. Issue 6. P. 714-720.

25. Samarskii A.A. The theory of Difference Schemes. NY, Marcel Dekker. 2001.

\section{For citation:}

Egorov A.A., Ayriyan A.S., Ayrjan E.A. Irregular liquid crystal waveguide structures: analysis of quasi-stationary fluctuations, power loss and statistical properties of irregularities. Zhurnal Radioelektroniki - Journal of Radio Electronics. 2020. No. 4. Available at http://jre.cplire.ru/jre/apr20/3/text.pdf. DOI 10.30898/1684-1719.2020.4.3 